\title{
Undernutrition, overweight and obesity among elderly living in communities in Africa: a systematic review
}

\author{
Gustave Mabiama $^{1,2,3}$, Dieudonné Adiogo $^{2}$, Thierno Millimono ${ }^{1}$, Philippe Fayemendy ${ }^{1,3}$, \\ Thibault Vernier ${ }^{3}$, Farid Boumediene ${ }^{1}$, Pierre-Marie Preux ${ }^{1}$, Jean-Claude Desport ${ }^{1,3}$ and \\ Pierre Jésus ${ }^{1,3}$ \\ ${ }^{1}$ Inserm U1094, Univ. Limoges, CHU Limoges, IRD, U1094, Tropical Neuroepidemiology, Institute of Epidemiology \\ and Tropical Neurology, GEIST, Limoges, France, \\ ${ }^{2}$ Laboratory of Microbiology, Immunology, Hematology and Morphological Sciences (LMIHSM), Doctoral Training \\ Unit of Health Sciences (UFD-SCS), Doctoral School, University of Douala, P.O. Box 2701, Douala, Cameroon". and \\ ${ }^{3}$ Health Network of the Nouvelle Aquitaine Regional Health Agency Limousin Nutrition (LINUT), Isle, France
}

Introduction Several studies in Africa and elsewhere have noted undernutrition, overweight and obesity among elderly people (1). African data were scattered and have not yet been subjected to a structured review. The objective of this study was to review studies on nutritional status (undernutrition, overweight, obesity) among elderly people living in communities in Africa, using Body Mass Index (BMI) and Mid-Upper Arm Circumference (MUAC) as tools. Methods Literature was extracted from Web of Science, GoogleScholar, PubMed, Research4life, AJOL and Scopus database using the following search terms: Africa/each of its country, older adults, elderly, nutrition/undernutrition/ overweight/obesity. Studies addressing nutritional status among the elderly (regardless of age) in Africa, irrespective of geographical area were included. Studies conducted in hospitals/nursing homes, using assessment tools other than BMI and MUAC, with insufficient information on methodology or with racial or gender discrimination were not included. The NEWCASTLE-OTTAWA QUALITY ASSESSMENT SCALE (NOQAS-adapted for cross sectional studies) was used to assess the quality of the articles. The four-stage review included 43 studies conducted in 17 countries covering almost all socio-geographical areas of the African continent. The systematic search was performed up to 25th November 2020. Two main types of studies (cross-sectional and prospective) were identified, conducted in rural and/or urban areas, with a sample between 62 and 2091, whose age was $\geq 55$ years. For each country, the average prevalence (undernutrition/overweight/obesity) and correlation with Human Development Index (HDI) of study period/publication was calculated. This systematic review was registered to PROSPERO under number CRD42021216268(2).

Results All studies were of fairly good quality with scores ranging from 6 to $9 / 10$. MUAC was exclusively used in two $(4.7 \%)$ of 43 studies and its data made less usable when BMI was used by $100 \%$. Using BMI, overall undernutrition was $19.9 \%$ in the continent, high in Central African Republic $(33.4 \%)$ and low in South Africa (3.4\%). Overweight (14.1\%) was high in South Africa (27.6\%) and low in Ethiopia (3.1\%) when obesity (12.8\%) was high in South Africa (50.4\%) and low in Tanzania (0.7\%). No correlation was found between undernutrition and the HDI $(r=-0.326, p=0.235)$. Overweight and obesity were respectively and positively correlated with HDI $(r=0.748$, $\mathrm{p}=0.003 ; \mathrm{r}=0.691, \mathrm{p}=0.004$ ). The sampling techniques were diverse and not always appropriate.

Conclusion Nearly one in five people in Africa is undernourished and about $30 \%$ are overweight or obese. This should be a challenge to African authorities, especially since excess weight could be a problem in the future due to the nutritional transition. Nevertheless, there is an urgent need of complementary studies more accurate and robust.

\section{Acknowledgements}

We would like to thank the ALAIR-Limousin Association and Health Network of the Nouvelle Aquitaine Regional Health Agency Limousin Nutrition (LINUT) for their financial support and the following members of the Groupe de Recherche sur le Vieillissement au Cameroon (GRVC) for facilitating and/or contributing to the data collection: Dr (Mrs) Véronique Priscille Nguiamba, Mrs Aurélie Michelle Mimba Mengue, Nadège Mayan Matouki, Edwige Laure Nzié, Cécile Nadège Kingué Ekamé, Didja Wassah, Annie Gaëlle Dekembaye, Brigitte Floriane Ombous, Anne Marie Ngo Manyim Ma Mayada, and Mr François Etotogo, Thierry Roland Njille Ehawa, Samaki Charles Yaya, Maurice Menzo, Lionel Wilfried Mbono Mbono; Dr Julien Magne for statistic guidance, as well as the elderly and their legal representatives.

\section{References}

1. Kimokoti RW \& Hamer DH (2008) Nutrition, Health and aging in sub-saharan Africa. Nutr Rev 66(11), 611-623.

2. Mabiama G, Millimono T, Adiogo D, et al. (2021) Undernutrition, overweight and obesity among the elderly living in communities in Africa: a systematic review. PROSPERO CRD42021216268, https://www.crd.york.ac.uk/prospero/display_record.php?ID=CRD42021216268 